\title{
Ocorrência de auto-incompatibilidade homomórfica em Mitracarpus longicalyx E. B. Souza \& M. F. Sales (Rubiaceae - Spermacoceae) ${ }^{1}$
}

\author{
ELNATAN B. DE SOUZA ${ }^{2,3,5}$, CRISTIANA B. N. COSTA ${ }^{3}$ e EDUARDO L. BORBA ${ }^{4}$
}

(recebido: 10 de agosto de 2006; aceito: 26 de abril de 2007)

\begin{abstract}
Occurrence of homomorphic self-incompatibility in Mitracarpus longicalyx E. B. Souza \& M. F. Sales (Rubiaceae - Spermacoceae)). The breeding system of Mitracarpus longicalyx was studied from September to December 2004 at Feira de Santana, BA, Brazil. This species is an annual herb found in the "caatinga" of northeastern Brazil, flowering between June and December. The inflorescences are glomerules with ca. 90 flowers. Each flower has a white 4-5 mm long hypocrateriform corolla. The flowers are protandrous with a three days long staminate phase followed by a five days long pistilate phase. No overlapping between phases was observed within a flower, but flowers in different phases appear in the same inflorescence. M. longycalyx is psycophilous species, pollinated by Hemiargus hanno hanno (Stoll, 1790) (Lycaenidae). Hand-pollination tests indicated that $M$. longicalyx is a self-incompatible, non-agamospermous species, with high fruit set under natural conditions (97.8\%) in the studied population; fruit set was also high (82.3\%) with hand cross-pollinations but geitonogamous hand pollination gave zero fruit set. Out-crossed pollen tubes reached the ovary within $24 \mathrm{~h}$ after pollination, while selfed pollen grains failed to germinate or selfed pollen tubes failed to penetrate the stigmatic papillae. The results indicate that M. longicalyx has homomorphic self-incompatibility, possibly of the sporophytic type, in addition to protandry. Homomorphic self-incompatibility is very rare in the Rubiaceae, a family with abundant occurrence of heteromorphic self-incompatibility.
\end{abstract}

Key words - breeding system, Mitracarpus, protandry, Rubiaceae, self-incompatibility

RESUMO -(Ocorrência de auto-incompatibilidade homomórfica em Mitracarpus longicalyx E. B. Souza \& M. F. Sales (Rubiaceae - Spermacoceae)). O estudo da biologia reprodutiva de Mitracarpus longicalyx E.B. Souza \& M.F. Sales foi realizado durante os meses de setembro a dezembro de 2004, em Feira de Santana, BA, Brasil. Esta espécie é uma erva anual ocorrente em áreas de caatinga, florescendo de junho a dezembro. As inflorescências são glomérulos com 90 flores em média, que apresentam corola hipocrateriforme, medindo 4-5 mm de comprimento e coloração branca, sendo visitadas e polinizadas por borboletas da espécie Hemiargus hanno hanno (Stoll, 1790) (Lycaenidae). As flores são protândricas, com duração da fase estaminada de três dias e da fase pistilada de cinco dias. Não ocorre sobreposição das duas fases na mesma flor, mas podem ocorrer flores em diferentes fases na mesma inflorescência. Polinizações experimentais indicaram que M. longicalyx é uma espécie autoincompatível não agamospérmica, com produção natural de frutos elevada $(97,8 \%)$ na população estudada. A frutificação também foi elevada nas polinizações cruzadas manuais $(82,3 \%)$, não ocorrendo frutificação nas polinizações manuais geitonogâmicas. Tubos polínicos provenientes de polinizações cruzadas alcançaram o ovário dentro de 24 horas, enquanto que grãos de pólen provenientes de autopolinização não germinaram ou, quando germinaram, não penetraram as papilas estigmáticas. Os resultados indicam que M. longicalyx possui auto-incompatibilidade homomórfica, possivelmente do tipo esporofítica, atuando em adição à protandria no impedimento da autofertilização. Auto-incompatibilidade homomórfica é muito rara nas Rubiaceae, uma família com abundante ocorrência de auto-incompatibilidade heteromórfica.

Palavras-chave - auto-incompatibilidade, Mitracarpus, protandria, Rubiaceae, sistemas de reprodução

\section{Introdução}

A família Rubiaceae possui distribuição cosmopolita, com maior riqueza na região tropical,

1. Parte da tese de doutorado do primeiro autor, Programa de Pós-Graduação em Botânica da Universidade Estadual de Feira de Santana.

2. Universidade Estadual Vale do Acaraú, Coordenação de Biologia, 62040-370 Sobral, CE, Brasil.

3. Universidade Estadual de Feira de Santana, Departamento de Ciências Biológicas, Km 03 - BR 116, 44031-460 Feira de Santana, BA, Brasil.

4. Universidade Federal de Minas Gerais, Instituto de Ciências Biológicas, Departamento de Botânica, Caixa Postal 486, 31270-901 Belo Horizonte, MG, Brasil.

5. Autor para correspondência: elbezsouza@yahoo.com.br abrangendo cerca de 12.000 espécies e 650 gêneros (Delprete 1999). Quanto à biologia reprodutiva, Robbrecht (1988) reconheceu três complexos de estratégias reprodutivas comuns entre as Rubiaceae que favoreceriam a alogamia: apresentação secundária de pólen, heterostilia e dioicia. A família possui o maior número de espécies heterostílicas dentre as angiospermas que apresentam esse tipo de polimorfismo (Barret 1992). Desta forma, a maioria dos trabalhos sobre a biologia reprodutiva de espécies de Rubiaceae tem sido realizada com espécies heterostílicas (Bawa \& Beach 1983, Pérez-Nasser et al. 1993, Richards \& Koptur 1993, Beliveau \& Wyatt 1999, Naiki \& Kato 1999, Castro \& Oliveira 2002, 
Teixeira \& Machado 2004a, b), com poucos estudos sendo realizados com espécies não heteromórficas. Em revisões realizadas por Gibbs (1990) e Jaimes \& Ramírez (1998), o único mecanismo de autoincompatibilidade encontrado nas Rubiaceae neotropicais é o do tipo heteromórfico e o primeiro autor sugere que maior atenção deveria ser dada ao estudo dos sistemas de reprodução em taxa homomórficos. Sistema de auto-incompatibilidade homomórfica gametofítica é referido raramente para algumas espécies da família, como em Coffea canephora Pierre (Devreux et al. 1959), Cephalanthus occidentalis L. (Imbert \& Richards 1993) e Manettia cordifolia Mart. (Consolaro et al. 2005).

O gênero Mitracarpus possui cerca de 40 espécies na América tropical (Souza \& Sales 2001), e registros sobre o sistema de reprodução no gênero são desconhecidos, sendo que mesmo informações sobre mecanismos de reprodução em gêneros afins são muito escassos. Mitracarpus longicalyx E. B. Souza \& M. F. Sales (Rubiaceae - Spermacoceae) é uma erva anual com distribuição circunscrita ao semi-árido brasileiro, ocorrendo em áreas de caatinga sobre solos de cobertura arenosa, ácidos e de baixa fertilidade (Souza \& Sales 2001), e suas flores não possuem indícios de serem heteromórficas. Dessein (2003) relatou a mesma dificuldade no seu estudo das Spermacoce L. africanas, e para toda a tribo Spermacoceae sensu stricto é também bastante escasso o conhecimento sobre os sistemas de reprodução, especialmente sobre a ocorrência de autoincompatibilidade, porém havendo várias menções de ocorrência de heterostilia (Robbrecht 1988), em especial para o gênero Galianthe (Cabral 1991, Cabral $\&$ Bacigalupo 1997).

O objetivo deste trabalho foi descrever o sistema de reprodução de $M$. longicalyx em uma população natural ocorrendo em área de caatinga no Nordeste do Brasil, determinando qual o tipo de estratégia reprodutiva está operando nesta espécie.

\section{Material e métodos}

O estudo foi realizado em área de caatinga no município de Feira de Santana, Bahia, 12 $11^{\circ} 54^{\prime \prime}$ 'S e $38^{\circ} 58^{\prime} 06^{\prime \prime}$ W, a cerca de $230 \mathrm{~m}$ de altitude, durante os meses de setembro e dezembro de 2004. O município possui clima semi-árido, e encontra-se situado em uma zona de planície, entre o Recôncavo e os tabuleiros, apresentando solos do tipo planossolo, podzólico, eutrófico e litólico. A vegetação típica é de caatinga, com fragmentos de matas secas (Floresta Estacional Decidual) e apresentando áreas com elementos da flora do cerrado (SEI 2004).

Para a realização das polinizações experimentais e observações, 13 indivíduos foram marcados. Foram realizadas observações sobre a morfologia floral, número de flores por inflorescência, duração da flor, coloração e acompanhamento do horário de antese. A receptividade estigmática foi verificada através de atividade de peroxidase (Kearns \& Inouye 1993), entre $8 \mathrm{~h} 00$ e $12 \mathrm{~h} 00$, e a disponibilidade polínica foi analisada mediante toque nas anteras e conseqüente liberação do pólen. As flores utilizadas para análise da morfologia foram conservadas em etanol a $70 \%$. Adicionalmente, os visitantes florais foram observados entre $8 \mathrm{~h} 00$ e $18 \mathrm{~h} 00$, durante dez dias, em um total de 30 horas de observação. Os polinizadores foram determinados com base na freqüência das visitas e no comportamento.

As polinizações manuais foram realizadas em flores em fase feminina de sete indivíduos dos treze selecionados inicialmente, sempre no período de $9 \mathrm{~h} 30$ a $11 \mathrm{~h} 30$. As inflorescências selecionadas para as polinizações foram previamente ensacadas com tule. Em cada inflorescência, as flores foram selecionadas em pré-antese e não foram emasculadas. Todas as demais flores do glomérulo foram removidas, uma vez que o arranjo floral fortemente congesto e o tamanho diminuto das flores dificultavam a execução das polinizações e a análise dos dados. A transferência do pólen foi efetuada movendo as anteras diretamente sobre o estigma receptivo, usando para essa operação uma pinça para segurar a corola da flor doadora. As polinizações cruzadas foram sempre realizadas com pólen proveniente de três outros indivíduos situados a pelo menos $100 \mathrm{~m}$ de distância da planta receptora, sendo utilizadas cinco plantas receptoras de pólen. A frutificação sob condições naturais (polinização aberta) foi avaliada em 46 flores previamente marcadas. Ocorrência de frutificação por agamospermia e/ou autopolinização espontânea foi avaliada em flores ensacadas e não manipuladas de quatro indivíduos.

A germinação dos grãos de pólen e o crescimento dos tubos polínicos nos tratamentos de autopolinização e polinização cruzada foram investigados usando microscopia de epifluorescência em pistilos fixados em FAA 50\%, em intervalos de 24, 48 e $72 \mathrm{~h}$. Os pistilos foram tratados com solução de $\mathrm{NaOH} 10 \mathrm{~N}$ em estufa a $60^{\circ} \mathrm{C}$ por 15 minutos, e corados com azul de anilina (modificado de Martin 1959). Espécimes da população foram depositados no acervo do herbário HUEFS (E.B. Souza 959, 964, 971).

\section{Resultados}

As flores de Mitracarpus longicalyx E. B. Souza \& M. F. Sales ocorrem em glomérulos compactos, 
congestos, axilares e terminais, com em média 90 flores $(n=10)$. As flores têm ovário ínfero, cálice tetralobado e corola hipocrateriforme, branca, com lobos ovaltriangulares, de $4,5 \mathrm{~mm}$ comprimento em média $(n=$ $60)$, e estilete de $4,7 \mathrm{~mm}$ comprimento $(n=10)$ (figuras 1-6). As flores se abrem por volta das $8 \mathrm{~h} 00$. M. longicalyx é uma espécie protândrica, com a oferta de pólen precedendo em três dias a receptividade estigmática. Em geral, 48 horas após o início da antese resta pouco pólen nas anteras, período que se inicia o alongamento do estilete (figura 3 ). A fase feminina pode durar em média cinco dias, e durante esse período os lobos do estilete começam a se encurvar e fenecer (figura 4-6). Apesar da separação temporal das fases nas flores, podem ocorrer flores em diferentes fases na mesma inflorescência (figura 1). Além da forte dicogamia, as flores de $M$. longicalyx são fortemente hercogâmicas (figuras 1-6).

Não ocorreu frutificação em autopolinização manual ou nas flores ensacadas não manipuladas. Por outro lado, houve uma elevada frutificação nas polinizações cruzadas experimentais e em polinização aberta, indicando que $M$. longicalyx é uma espécie autoincompatível (tabela 1). Os grãos de pólen provenientes de autopolinização geralmente não germinaram, ou, quando germinaram, não penetraram as papilas estigmáticas (figuras 7-9). Por outro lado, foi observada elevada germinação de grãos de pólen em polinizações cruzadas após 24 horas (figura 10), tendo os tubos polínicos alcançados o ovário e penetrados os óvulos após este período (figuras 11-12).

No período de observação, a borboleta Hemiargus hanno hanno (Stoll, 1790) (Lycaenidae, Polyommatinae), espécie comum em áreas abertas onde $M$. longicalyx ocorre, foi o único visitante, e considerado o polinizador efetivo. Essas pequenas borboletas visitam sistematicamente várias flores de uma mesma inflorescência introduzindo sua probóscide no tubo da corola e tocando nas estruturas reprodutivas, realizando a polinização.

\section{Discussão}

De acordo com Lloyd \& Webb (1986) a manutenção da protandria em uma espécie autoincompatível pode indicar uma seleção para as seguintes condições: 1) apresentação prolongada de pólen; 2) evitar a interferência pólen-estigma na mesma flor; e 3) oferecer posição ótima para doação e recepção de pólen. Aparentemente, a manutenção da protandria em M. longicalyx está relacionada à segunda condição, evitando perda de pólen em polinizações incompatíveis. A protandria pode também estar relacionada ao mecanismo de apresentação secundária de pólen em muitos grupos de Rubiaceae, como ocorre com Gardenieae (Robbrecht 1988). Em Cephalanthus occidentalis L. a protandria foi associada a um sistema de auto-incompatibilidade homomórfico possivelmente gametofítico (Imbert \& Richards 1993). A protandria observada em M. longicalix é muito rara em Rubiaceae, sendo muito similar ao encontrado em Putoria calabrica (L.f.) DC., inclusive em relação ao polimorfismo da corola durante o desenvolvimento (Ortiz et al. 2000). Nessa espécie, Ortiz et al. (2000) consideram a autopolinização improvável, mas a geitonogamia freqüente, uma vez que, assim como em $M$. longicalix, existem várias flores em ambas as fases no mesmo indivíduo, acarretando em autogamia uma vez que $P$. calabrica, diferente de $M$. longicalyx, é autocompatível. A estratégia reprodutiva encontrada em $M$. longicalyx é também semelhante àquela encontrada em Galium mucroniferum Sond. e G. subvillosum Sond., onde o grão de pólen é oferecido logo após a antese e a fase feminina estabelecida entre quatro e seis dias mais tarde, quando o estilete já tinha alcançado seu comprimento máximo e os estigmas tornados receptivos (Puff 1978). Mais comumente, protandria em Rubiaceae está associada à apresentação secundária de pólen no estilete (Robbrecht 1988). Em espécies de Rubiaceae autocompatíveis, como Ferdinandusa speciosa Pohl. (Castro \& Oliveira 2001), a protandria pode favorecer a alogamia.

Segundo Richards (1997), a hercogamia é um fenômeno especialmente comum em plantas autoincompatíveis, separando as funções masculina e feminina no espaço e propiciando o transporte de pólen entre os indivíduos e populações. A hercogamia é, portanto, vantajosa por permitir a alogamia e por reduzir a obstrução dos estigmas com pólen de polinizações incompatíveis.

A auto-incompatibilidade em $M$. longicalyx está associada a um sítio de inibição estigmático, similar ao observado em Coffea (Devreux et al. 1959), mas diferente do que ocorre em Cephalanthus occidentalis L. (Imbert \& Richards 1993) e Manettia cordifolia Mart. (Consolaro et al. 2005), onde a autoincompatibilidade está caracterizada por um sítio de inibição no estilete. Auto-incompatibilidade homomórfica com formação de tubo polínico, mas sem local preciso de inibição, também sido observada em outros gêneros de Rubiaceae, como em Hamelia, Posoqueria e Warszewiczia (Bawa \& Beach 1983). Este sítio de 

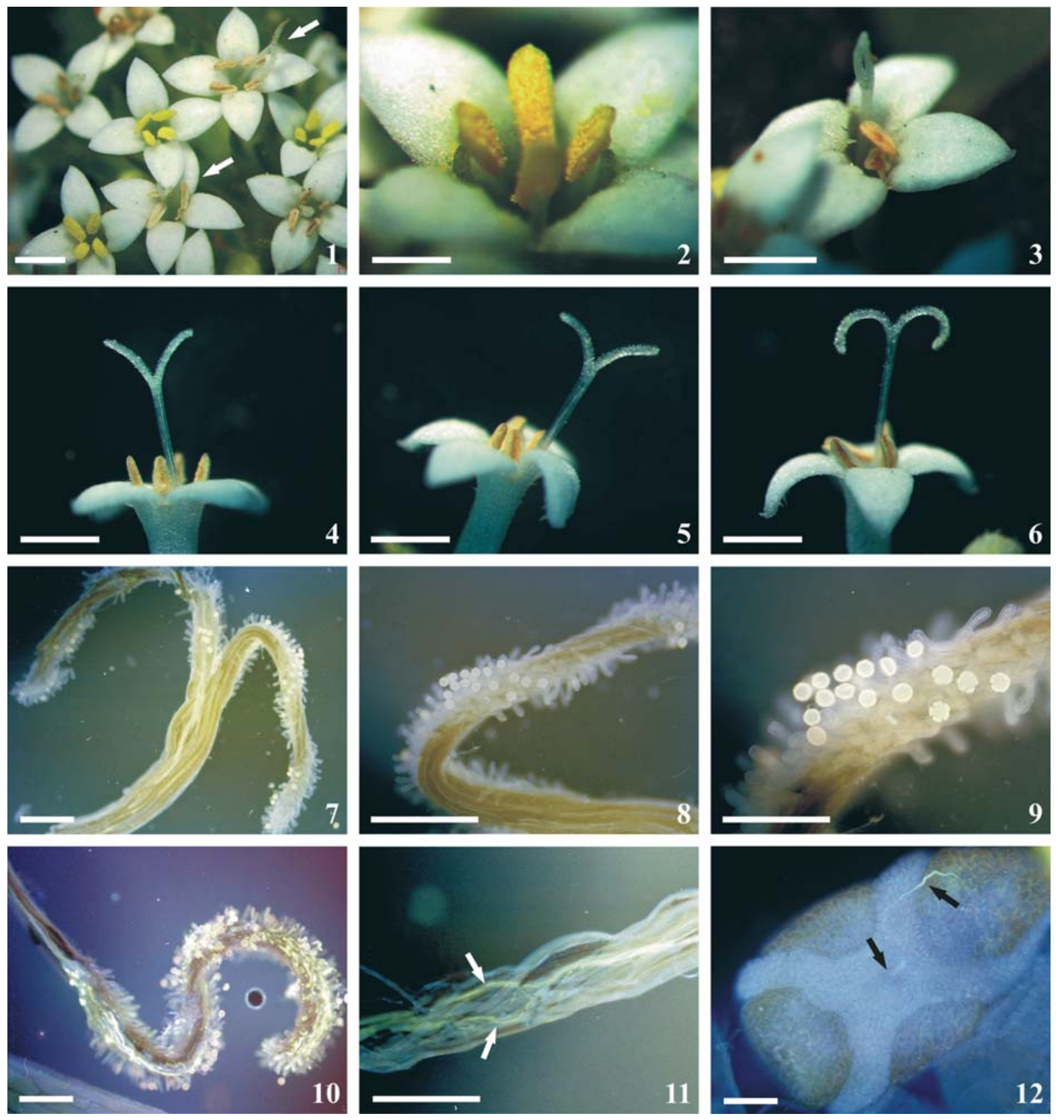

Figuras 1-12. Mitracarpus longicalyx E. B. Souza \& M. F. Sales (Rubiaceae). 1-6. Flores 1. Parte da inflorescência; note a presença de flores em fase pistilada (seta) e em fase estaminada (apresentando anteras amarelas). 2. Flor em fase masculina. 3. Início do alongamento do estilete (algumas anteras foram removidas). 4-6. Desenvolvimento do estilete e abertura dos ramos estigmáticos. 7-12. Germinação dos grãos de pólen e crescimento do tubo polínico em microscopia de epifluorescência. 7. Ramos estigmáticos após autopolinização. 8-9. Aspecto das papilas e do ramo estigmático com grãos de pólen não-germinantes em autopolinização. 10. Estigma mostrando a germinação dos grãos de pólen em polinização cruzada. 11. Crescimento do tubo polínico no estilete em polinização cruzada (setas). 12. Tubo polínico penetrando o óvulo em polinização cruzada (setas). Barras $=0,5 \mathrm{~mm}(1-2) ; 1,0 \mathrm{~mm}(3-6) ; 0,2 \mathrm{~mm}(7-8,10-12) ; 0,1 \mathrm{~mm}(9)$.

Figures 1-12. Mitracarpus longicalyx E.B. Souza \& M.F. Sales (Rubiaceae). 1-6. Flowers 1. Part of inflorescence; notice the occurrence of flowers in pistillate phase (arrow) and staminate phase (bearing yellow anthers). 2. Flower in staminate phase. 3. Beginning of the growth of the style (some anthers were removed). 4-6. Development of the style and opening of stigma branches. 7-12. Pollen grain germination and pollen tube growth in epifluorescence microscopy. 7. Stigma branches after selfpollination. 8-9. Aspects of stigma papillae and stigma branch with selfed pollen grains in self-pollination. 10. Stigma showing pollen grain germination in outcrossing. 11. Crossed pollen tube growth in the style (arrows). 12. Crossed pollen tube reaching the ovule (arrows). Bars $=0.5 \mathrm{~mm}(1-2) ; 1.0 \mathrm{~mm}(3-6) ; 0.2 \mathrm{~mm}(7-8,10-12) ; 0.1 \mathrm{~mm}(9)$. 
inibição encontrado em $M$. longicalyx é característico do sistema homomórfico esporofítico, mecanismo menos comum entre as angiospermas (Gibbs 1990, Richards 1997). Porém, a presença de sistema de autoincompatibilidade do tipo gametofítico associada à inibição estigmática tem sido confirmada para espécies de Coffea (Lashermes et al. 1996) e para outras angiospermas (Richards 1997). Acreditamos que os cruzamentos de indivíduos como efetuado neste estudo tornam pouco plausível o cenário de ocorrência de autoincompatibilidade dialélica, como observado em espécies heterostílicas, sendo mais provável nessa espécie a ocorrência de auto-incompatibilidade homomórfica multialélica. Porém, ainda necessita ser estabelecido se esta é do tipo gametofítico ou esporofítico.

Como ressaltado por Gibbs (1990), é muito importante a ampliação dos conhecimentos sobre os sistemas de reprodução em outras espécies de Rubiaceae com auto-incompatibilidade homomórfica, sendo inclusive necessária a realização de cruzamentos dialélicos para determinação do tipo de incompatibilidade (esporofítica ou gametofítica) presente e, eventualmente, reavaliar a ocorrência destes dois sistemas na família. Empregando essa abordagem, Talavera et al. (2001) conseguiram determinar a ocorrência de auto-incompatibilidade homomórfica gametofítica controlada por um único locus polimórfico em Anagallis monelli L.(Primulaceae), espécie de uma família predominantemente com ocorrência de autoincompatibilidade heteromórfica. A ausência de espécies comprovadamente com autoincompatibilidade homomórfica esporofítica nas principais famílias portadoras de autoincompatibilidade heteromórfica (tipicamente sob controle esporofítico) é um dos principais argumentos contrários à hipótese da evolução desta última a partir da primeira (Ganders 1979, Gibbs 1986). Por outro lado, ocorrência de auto-incompatibilidade heteromórfica e auto-incompatibilidade gametofítica em uma mesma família é comum (de Nettancourt 1977).

Entre os sistemas de auto-incompatibilidade conhecidos para Rubiaceae, o mais amplamente distribuído na família é o sistema heteromórfico heterostílico. Autocompatibilidade também ocorre na família (Jaimes \& Ramírez 1998), inclusive em espécies homostílicas pertencentes a gêneros tipicamente com espécies auto-incompatíveis heterostílicas, como observado em Palicourea macrobotrys Ruiz \& Pavon (Coelho \& Barbosa 2003). Na tribo Spermacoceae, como descrito por Dessein (2003), o sistema heteromórfico é o mais conhecido, sendo registrado para os gêneros Galianthe (Cabral 1991, Cabral \& Bacigalupo 1997), Schwendenera (Schumann 1889), Psyllocarpus (Kirkbride 1979) e para os gêneros caribenhos monotípicos Tobagoa e Tortuella (Dessein 2003). $\mathrm{O}$ estudo com $M$. longicalyx representa o primeiro registro de auto-incompatibilidade homomórfica para a tribo Spermacoceae sensu stricto, possivelmente do tipo esporofítica. São necessários, contudo, mais estudos dentro da tribo Spermacoceae e no próprio gênero para uma compreensão mais ampla da evolução da auto-incompatibilidade e dos mecanismos que estão atuando sobre os processos reprodutivos deste grupo.

Agradecimentos - Ao Dr. Marcelo Duarte, à Dra. Favízia F. Oliveira e ao Ivan F. Castro pela identificação do espécime de borboleta, à Carlianne O. C. Ramos e à Vânia M. Passos pela colaboração no trabalho de campo e laboratório e pelas críticas e sugestões, à Renata Dias Souza pelo auxílio no tratamento dos negativos, a dois assessores anônimos pelas críticas e sugestões, à Fundação Cearense de Apoio ao Desenvolvimento Científico e Tecnológico (FUNCAP) pela bolsa de doutorado do primeiro autor e ao CNPq pela bolsa de produtividade em pesquisa concedida ao último autor (PQ2).

Tabela 1. Frutificação nos diferentes tratamentos de polinização experimental realizados em flores de Mitracarpus longicalyx E. B. Souza \& M. F. Sales (Rubiaceae) em área de caatinga no Município de Feira de Santana, BA, Brasil.

Table 1. Fruit set in different experimental pollination treatments carried out in flowers of Mitracarpus longicalyx E. B. Souza \& M. F. Sales (Rubiaceae) in a "caatinga" area in the municipality of Feira de Santana, BA, Brazil.

\begin{tabular}{lcccc}
\hline Tratamento & Flores & Indivíduos & Inflorescências & Frutificação (\%) \\
\hline Autopolinização induzida & 45 & 5 & 5 & 0 \\
Polinização cruzada induzida & 34 & 5 & 5 & 82,3 \\
Polinização natural (controle) & 46 & 2 & 2 & 97,8 \\
Autopolinização espontânea/Agamospermia & 154 & 4 & 4 & 0 \\
\hline
\end{tabular}




\section{Referências bibliográficas}

BARRETT, S.C.H. 1992. Heterostylous genetic polymorphisms: model systems for evolutionary analysis. In Evolution and function of heterostyly (S.C.H. Barrett, ed.). Springer-Verlag, Berlin, p.1-24.

BAWA, K.S. \& BEACH, J.H. 1983. Self-incompatibility systems in the Rubiaceae of a tropical lowland wet forest. American Journal of Botany 70:1281-1288.

BELIVEAU, B.D. \& WYATT, R. 1999. Compatibility relationships in distylous bluets: Houstonia serpyllifolia and $H$. longifolia (Rubiaceae). The American Midland Naturalist 141:217-226.

CABRAL, E.L. 1991. Rehabilitacion del género Galianthe (Rubiaceae). Boletín de la Sociedad Argentina de Botánica 27:225-249.

CABRAL, E.L. \& BACIGALUPO, N.M. 1997. Revisión del género Galianthe subg. Ebelia stat. nov. (Rubiaceae: Spermacoceae). Annals of the Missouri Botanical Garden 84:857-877.

CASTRO, C.C. \& OLIVEIRA, P.E.A.M. 2001. Reproductive biology of the protandrous Ferdinandusa speciosa Pohl (Rubiaceae) in Southeastern Brazil. Revista Brasileira de Botânica 24:167-172.

CASTRO, C.C. \& OLIVEIRA, P.E.A.M. 2002. Pollination biology of distylous Rubiaceae in the Atlantic Rain Forest, SE, Brazil. Plant Biology 4:640-646.

COELHO, C.P. \& BARBOSA, A.A.A. 2003. Biologia reprodutiva de Palicourea macrobotrys Ruiz \& Pavon (Rubiaceae): um possível caso de homostilia no gênero Palicourea Aubl. Revista Brasileira de Botânica 26:403-413.

CONSOLARO, H., SILVA, E.B. \& OLIVEIRA, P.E. 2005. Variação floral e biologia reprodutiva de Manettia cordifolia Mart. (Rubiaceae). Revista Brasileira de Botânica 28:85-94.

DELPRETE, P.G. 1999. Flora Neotropica. Monograph 77. Rondeletieae (Rubiaceae) - Part I. New York Botanical Garden, New York.

DE NETTANCOURT, D. 1977. Incompatibility in angiosperms. Springer-Verlag, Berlin.

DESSEIN, S. 2003. Systematic studies in the Spermacoceae (Rubiaceae). Tese de Doutorado, Institute of Botany and Microbiology, Leuven.

DEVREUX, M., VALLAEYS, G., POCHET, P. \& GILLES, A. 1959. Recherches sur l'autosterilité du caféier robusta (Coffea canephora Pierre). Publ. INEAC 78:1-44.

GANDERS, F.R. 1979. The biology of heterostyly. New Zealand Journal of Botany 17:607-635.

GIBBS, P.E. 1986. Do homomorphic and heteromorphic selfincompatibility systems have the same sporophytic mechanisms? Plant Systematics and Evolution 154:285323.

GIBBS, P.E. 1990. Self-incompatibility in flowering plants: a neotropical perspective. Revista Brasileira de Botânica 13:125-136.
IMBERT, F.M. \& RICHARDS, J.H. 1993. Protandry, incompatibility, and secondary pollen presentation in Cephalanthus occidentalis (Rubiaceae). American Journal of Botany 80:395-404.

JAIMES, I \& RAMÍREZ, N. 1998. Autoincompatibilidad, autogamia y agamospermia en angiospermas tropicales. Acta Biologica Venezuelica 18:59-80.

KEARNS, C.A. \& INOUYE, D.W. 1993. Techniques for pollination biologists. University Press of Colorado, Niwot.

KIRKBRIDE, J.H. 1979. Revision of the genus Psyllocarpus (Rubiaceae). Smithsonian Contributions to Botany 41:1-31.

LASHERMES, P., COUTURON, E., MOREAU, N., PAILLARD, M. \& LOUARN J. 1996. Inheritance and genetic mapping of self-incompatibility in Coffea canephora Pierre. Theoretical and Applied Genetics 93:458-462.

LLOYD, D.G. \& WEBB, C.J. 1986. The avoidance interference between the presentation of pollen and stigmas in angiosperms. I. Dichogamy. New Zealand Journal of Botany 24:135-162.

MARTIN, F.W. 1959. Staining and observing pollen tubes in the style by means of fluorescence. Stain Technology 34:125-128.

NAIKI, A. \& KATO, M. 1999. Pollination system and evolution of dioecy from distyly in Mussaenda parviflora (Rubiaceae). Plant Species Biology 14:217227.

ORTIZ, P.L., ARISTA, M. \& GIBBS, P.E. 2000. Pollination and breeding system of Putoria calabrica (Rubiaceae), a Mediterranean dwarf shrub. Plant Biology 2:325-330.

PÉREZ-NASSER, N., EGUIARTE, L.E. \& PIÑERO, D. 1993. Mating system and genetic structure of the distylous tropical tree Psychotria faxlucens (Rubiaceae). American Journal of Botany 80:45-52.

PUFF, C. 1978. The genus Gallium L. (Rubiaceae) in southern Africa. South African Journal of Botany 44:203-279.

RICHARDS, A.J. 1997. Plant breeding systems, $2^{\mathrm{a}}$ ed. George Allen \& Unwin, London.

RICHARDS, J.H. \& KOPTUR, S. 1993. Floral variation and distyly in Guettarda scabra (Rubiaceae). American Journal of Botany 80:31-40.

ROBBRECHT, E. 1988. Tropical woody Rubiaceae. Opera Botanica Belgica 1:1-271.

SCHUMANN, K. 1889. Rubiaceae. Schwendenera. In Flora brasiliensis (C.F.P.Martius \& A.G. Eichler, eds.). F. Fleischer, Lipsiae, v.6, pars 6, p.98-99.

SEI - Superintendência de Estudos Econômicos e Sociais da Bahia. 2004. http://www.sei.ba.gov.br (acesso em 01/12/ 2004).

SOUZA, E.B. \& SALES, M.F. 2001. Mitracarpus longicalyx (Rubiaceae, Spermacoceae), a new species from northeastern Brazil. Brittonia 53:482-486.

TALAVERA, S., GIBBS, P.E., FERNÁNDEZ-PIEDRA, M.P. \& ORTIZ-HERRERA, M.A. 2001. Genetic control of selfincompatibility in Anagallis monelli (Primulaceae: Myrsinaceae). Heredity 87:589-597. 
TEIXEIRA, L.A.G. \& MACHADO, I.C. 2004a. Sabicea cinerea Aubl. (Rubiaceae): distilia e polinização em um fragmento de Floresta Atlântica em Pernambuco, Nordeste do Brasil. Revista Brasileira de Botânica 27:193-204.
TEIXEIRA, L.A.G. \& MACHADO, I.C. 2004b. Biologia da polinização e sistema reprodutivo de Psychotria barbiflora DC. (Rubiaceae). Acta Botanica Brasilica 18:853-862. 